\title{
The Effect of Morning Stiffness Duration on the Definition of Clinically Inactive Disease in Juvenile Idiopathic Arthritis
}

\author{
Maddalena Allegra, Maria Francesca Gicchino, Gabriella Giancane, Alessandra Alongi, \\ Jessica Tibaldi, Marta Mazzoni, Alessandro Consolaro, and Angelo Ravelli
}

\begin{abstract}
Objective. To investigate the effect of morning stiffness (MS) on parent disease perception in children with juvenile idiopathic arthritis (JIA) with clinically inactive disease (CID).

Methods. We examined 652 visits in which patients fulfilled 2004 or 2011 Wallace criteria for CID. Parent-reported outcomes were compared among patients with no MS or with $\mathrm{MS}<$ or $\geq 15$ min.

Results. Among 652 visits with CID by 2004 criteria, no MS was reported in 554 visits (85\%), MS $<15 \mathrm{~min}$ in $53(8 \%)$, and $\mathrm{MS} \geq 15 \mathrm{~min}$ in $45(7 \%)$. The frequency of altered physical function, health-related quality of life and well-being, pain, and disease activity visual analog scales was proportionally greater in patients without MS than those with longer MS. The frequency of parent subjective rating of disease state as remission was $87.7 \%, 58 \%$, and $26.7 \%$ among patients with no MS, MS $<15 \mathrm{~min}$, and MS $\geq 15 \mathrm{~min}$, respectively.

Conclusion. Our results suggest that a change in 2011 CID criteria to require absence of MS should be considered. (First Release April 15 2020; J Rheumatol 2020;47:1238-41; doi:10.3899/jrheum.190890)
\end{abstract}

\section{Key Indexing Terms: \\ JUVENILE IDIOPATHIC ARTHRITIS REMISSION}

Morning stiffness (MS) is a major symptom of active disease in children with juvenile idiopathic arthritis (JIA), and may have a profound effect on physical function and health-related quality of life (HRQOL) ${ }^{1,2}$.

In the past 2 decades there have been remarkable advances in the management of JIA, comprising the advent of medications that are capable of inducing extended periods of complete disease quiescence. Owing to the key importance of MS on child well-being, it would be important to ascertain whether the achievement of clinical remission is accompanied by its abrogation.

Assessment of MS was not incorporated into the

From the Istituto Giannina Gaslini IRCCS, Genoa, Italy; Università degli Studi di Genova, Genoa, Italy; Sechenov First Moscow State Medical University, Moscow, Russia.

M. Allegra, MD, Research Fellow, Istituto Giannina Gaslini IRCCS; M.F. Gicchino, MD, Pediatric Resident, Istituto Giannina Gaslini IRCCS; G. Giancane, MD, PhD, Assistant Professor, Istituto Giannina Gaslini IRCCS, and Università degli Studi di Genova; A. Alongi, $M D, P h D$ Student, Istituto Giannina Gaslini IRCCS; J. Tibaldi, MD, PhD Student, Istituto Giannina Gaslini IRCCS; M. Mazzoni, MD, PhD Student, Istituto Giannina Gaslini IRCCS; A. Consolaro, MD, PhD, Assistant Professor, Istituto Giannina Gaslini IRCCS, and Università degli Studi di Genova; A. Ravelli, MD, Professor of Pediatrics, Istituto Giannina Gaslini IRCCS, and Università degli Studi di Genova, and Sechenov First Moscow State Medical University. Dr. Allegra and Dr. Gicchino contributed equally to this study.

Address correspondence to Prof. A. Ravelli, Clinica Pediatrica e Reumatologia, Istituto G. Gaslini, Via G. Gaslini 5, 16147 Genoa, Italy. E-mail: angeloravelli@gaslini.org

Accepted for publication October 23, 2019.

\section{MORNING STIFFNESS PEDIATRIC RHEUMATIC DISEASES}

preliminary criteria for clinically inactive disease (CID) in JIA (thereafter called "2004 CID criteria") 3 . By these criteria, CID requires the simultaneous presence of the following: (1) no active joints; (2) absence of systemic symptoms attributable to JIA; (3) absence of active uveitis; (4) normal erythrocyte sedimentation rate (ESR) and C-reactive protein (CRP); and (5) a physician's global assessment indicating no disease activity.

A 2011 revision of the 2004 CID criteria (thereafter called "2011 CID criteria") specified the definition of inactive uveitis, acknowledged that ESR or CRP can be elevated for reasons unrelated to JIA, and added the presence of MS lasting $\leq 15 \mathrm{~min}^{4}$. This cutoff was based on the belief that $\mathrm{MS} \leq 15$ min may represent damage from previous active disease or be due to reasons other than active inflammation. However, any cutoff is arbitrary, and it is unclear whether a duration of $\mathrm{MS} \leq 15 \mathrm{~min}$ is equivalent to no MS, particularly when it comes to parent-reported outcomes (PRO).

Our present study compared PRO of children with JIA who met 2004 or 2011 CID criteria and had no MS, MS $<15$ $\min$, or $\mathrm{MS} \geq 15 \mathrm{~min}$.

\section{MATERIALS AND METHODS}

Patient selection. Two datasets including 1208 children with $\mathrm{JIA}^{5}$ who underwent a total of 3380 visits were examined to identify all visits in which patients fulfilled the 2004 or 2011 criteria for CID. If a patient met CID criteria in more than 1 visit, only the first visit was retained. The first dataset (named EtICA dataset) included 422 patients treated with etanercept (ETN) who underwent a single cross-sectional visit ${ }^{6}$. The second dataset (named Gaslini dataset) was composed of 816 unselected patients followed at an authors' center between September 2013 and May 2018.

Personal non-commercial use only. The Journal of Rheumatology Copyright $($ C 2020. All rights reserved. 
The study protocol was approved by the ethics committee of Liguria, Genoa, Italy (session of June 18, 2018, meeting minutes no. 10/2018).

Clinical assessments. At each visit, the caring physician made a standardized joint examination ${ }^{7}$ and rated the overall disease activity on a 21-numbered circle visual analog scale $(0=$ no activity; $10=$ maximum activity $)^{8}$.

Before the physician visit, a parent (or guardian) completed the Italian-language parent proxy-report version of the Juvenile Arthritis Multidimensional Assessment Report (JAMAR) ${ }^{9}$. This questionnaire incorporates all main PRO, including physical function, HRQOL, overall well-being, pain, level of disease activity, MS, subjective assessment of disease status, and satisfaction with illness outcome.

Informed consent to original data collections was provided by parents/ guardians for all patients.

Assessment of MS. The JAMAR includes a question that asks the parent whether the child had joint stiffness upon waking up over the previous week, and is to be answered "yes" or "no." If the answer is yes, then the parent is asked how long the MS lasts by marking 1 of these intervals: $<15$ $\min ; 15-30 \mathrm{~min} ; 30 \mathrm{~min}-1 \mathrm{~h} ; 1-2 \mathrm{~h} ;>2 \mathrm{~h}$. For the purposes of the analysis, the time intervals were dichotomized as $<15 \mathrm{~min}$ or $\geq 15 \mathrm{~min}$.

Assessment of inactive disease. The presence of inactive disease was assessed by both the 2004 CID criteria ${ }^{3}$ and the 2011 CID criteria ${ }^{4}$ (see above). No imputation of missing data was performed. In case both ESR and CRP or ophthalmologic assessment were not available, the disease state was defined as CID if all remaining criteria were met.

\section{RESULTS}

A total of 652 visits in which patients met either 2004 or 2011 CID criteria, or both, were identified. The main clinical features of these patients are presented in Table 1. As expected, patients in the EtICA dataset, who were all treated with ETN, had a higher frequency of polyarthritis and extended oligoarthritis than those in the Gaslini dataset, which was composed of patients followed in routine care. They were also older and had longer disease duration than those in the Gaslini dataset. Because the data by level of MS were comparable between the 2 datasets (Supplementary Table 1, available from the authors on request), they were combined in the study analyses.
Among the 652 visits/patients with CID by 2004 or 2011 criteria, no MS was reported in 554 visits (85\%), MS $<15 \mathrm{~min}$ in $53(8 \%)$, and $\mathrm{MS} \geq 15 \mathrm{~min}$ in $45(7 \%)$. All 652 patients met the 2004 CID criteria, which do not include assessment of MS, whereas 45 patients $(6.9 \%)$ did not meet the 2011 CID criteria because of MS $\geq 15 \mathrm{~min}$.

The demographic and clinical features of patients with no MS and MS $<15$ or $\geq 15$ min were comparable (Table 2). As shown in Table 3, patients with MS of any duration had a higher frequency of abnormality of all PRO than patients with no MS. Patients with $\mathrm{MS} \geq 15 \mathrm{~min}$ had a higher frequency of abnormality in most PRO than patients who had MS $<15$ min.

The frequency of altered PRO by parent-assessed disease state and by presence and duration of MS in relation to parent-assessed disease state is presented in Supplementary Tables 2, 3, and 4 (available from the authors on request).

\section{DISCUSSION}

We found that $15 \%$ of patients meeting the 2004 CID criteria had MS and that $8.1 \%$ of patients meeting the 2011 CID criteria had MS $<15$ min. Patients with MS had worse PRO than those without MS. Most PRO listed more impairment in patients with $\mathrm{MS} \geq 15 \mathrm{~min}$ than in patients with $\mathrm{MS}<15$ min. The observed disparities did not depend on differences in disease characteristics and severity. These findings indicate that the presence of MS is associated with worse parent perception of health and disease status of children with JIA who are classified as having CID by formal definitions.

As emphasized previously ${ }^{10}$, a major problem with 2004 CID criteria is that they are based only on physician-centered measures and an acute-phase reactant, and neglect PRO. Integration of patient and parent perspective into the clinical evaluation is important because achievement

Table 1. Demographic features and ILAR categories of study patients.

\begin{tabular}{lcccc}
\hline Variables & $\begin{array}{c}\text { All Patients, } \\
\mathrm{n}=652\end{array}$ & $\begin{array}{c}\text { EtICA Dataset, } \\
\mathrm{n}=199\end{array}$ & $\begin{array}{c}\text { Gaslini Dataset, } \\
\mathrm{n}=453\end{array}$ & $\mathrm{p}$ \\
\hline Females & $510(78.2)$ & $152(76.4)$ & $358(79.0)$ & 0.51 \\
ILAR category & & & & $<0.001$ \\
Systemic arthritis & $24(3.7)$ & $4(2.0)$ & $20(4.4)$ & \\
Persistent oligoarthritis & $250(38.3)$ & $34(17.1)$ & $216(47.7)$ & \\
RF-negative polyarthritis & $130(19.9)$ & $60(30.2)$ & $70(15.5)$ & \\
Extended oligoarthritis & $185(28.4)$ & $73(36.7)$ & $112(24.7)$ & \\
RF-positive polyarthritis & $11(1.7)$ & $6(3.0)$ & $5(1.1)$ & \\
Psoriatic arthritis & $16(2.5)$ & $8(4.0)$ & $8(1.8)$ & \\
Enthesitis-related arthritis & $19(2.9)$ & $12(6.0)$ & $7(1.5)$ & \\
Undifferentiated arthritis & $17(2.6)$ & $2(1.0)$ & $15(3.3)$ & \\
Median (IQR) disease duration, yrs & $4.7(2.3-8.7)$ & $7.1(4.1-11.1)$ & $3.8(1.7-7.4)$ & $<0.001$ \\
Median (IQR) age at visit, yrs & $9.7(5.4-13.9)$ & $13.2(8.4-16.4)$ & $8.3(4.6-12.3)$ & $<0.001$ \\
Median (IQR) age at disease onset, yrs & $2.9(1.8-5.9)$ & $3.3(1.9-7.4)$ & $2.7(1.7-4.8)$ & 0.005 \\
\hline
\end{tabular}

Data are n (\%) unless otherwise indicated. ILAR: International League of Associations of Rheumatology; RF: rheumatoid factor; IQR: interquartile range.

Personal non-commercial use only. The Journal of Rheumatology Copyright $\subset$ 2020. All rights reserved. 
Table 2. Demographic and clinical features by presence and duration of morning stiffness.

\begin{tabular}{lcccc}
\hline Variables & \multicolumn{3}{c}{ Patients Meeting 2004 CID Criteria } & \\
& Patients Meeting 2011 CID Criteria & & $\mathrm{p}$ \\
& No MS, & MS < 15 min, & MS $\geq 15$ min, & \\
& $\mathrm{n}=554$ & $\mathrm{n}=53$ & $\mathrm{n}=45$ & \\
\hline Females & $431(77.8)$ & $43(71.1)$ & $36(80.0)$ & 0.82 \\
ILAR category & & & & 0.96 \\
Systemic arthritis & $20(3.6)$ & $2(3.8)$ & $2(4.4)$ & \\
Persistent oligoarthritis & $219(39.5)$ & $16(30.2)$ & $15(33.3)$ & \\
RF-negative polyarthritis & $153(27.6)$ & $19(35.8)$ & $13(28.9)$ & \\
Extended oligoarthritis & $109(19.7)$ & $10(18.9)$ & $11(24.4)$ & \\
RF-positive polyarthritis & $10(1.8)$ & $0(0.0)$ & $1(2.2)$ & \\
Psoriatic arthritis & $14(2.5)$ & $1(1.9)$ & $1(2.2)$ & \\
Enthesitis-related arthritis & $15(2.7)$ & $3(5.7)$ & $1(2.2)$ & \\
Undifferentiated arthritis & $14(2.5)$ & $2(3.8)$ & $1(2.2)$ & \\
Median (IQR) disease duration, yrs & $4.6(2.3-8.8)$ & $3.9(1.9-7.3)$ & $6.7(3.1-9.1)$ & 0.17 \\
Median (IQR) age at visit, yrs & $9.7(5.5-13.8)$ & $8.3(4.8-11.8)$ & $10.6(6.9-15.2)$ & 0.23 \\
Median (IQR) age at disease onset, yrs & $2.8(1.7-5.8)$ & $3.2(1.9-5.5)$ & $2.9(1.9-7.0)$ & 0.55 \\
Patients with no. tender joints $>0$ & $35(6.3)$ & $3(5.7)$ & $5(11.1)$ & 0.442 \\
Patients with no. restricted joints $>0$ & $111(20.0)$ & $8(15.1)$ & $6(13.3)$ & 0.401 \\
\hline
\end{tabular}

Data are n (\%) unless otherwise indicated. CID: clinically inactive disease; MS: morning stiffness; ILAR: International League of Associations of Rheumatology; RF: rheumatoid factor; IQR: interquartile range.

Table 3. Parent-reported outcomes by presence and duration of morning stiffness.

\begin{tabular}{|c|c|c|c|c|}
\hline \multirow[t]{2}{*}{ Variables } & \multicolumn{3}{|c|}{$\begin{array}{l}\text { Patients Meeting } 2004 \text { CID Criteria } \\
\text { Patients Meeting } 2011 \text { CID Criteria }\end{array}$} & \multirow[t]{2}{*}{$\mathrm{p}$} \\
\hline & $\begin{array}{l}\text { No MS, } \\
\mathrm{n}=554\end{array}$ & $\begin{array}{c}\mathrm{MS}<15 \min \\
\mathrm{n}=53\end{array}$ & $\begin{aligned} \mathrm{MS} & \geq 15 \mathrm{~min} \\
\mathrm{n} & =45\end{aligned}$ & \\
\hline Patients with physical function score ${ }^{a}>0$ & $104(18.8)$ & $24(45.3)$ & $35(77.8)$ & $<0.001$ \\
\hline \multicolumn{5}{|c|}{ Patients with HRQOL PhH score ${ }^{b}>1$ SD above } \\
\hline the mean of healthy children & $57 / 550(10.4)$ & $22 / 52(42.3)$ & $35 / 44(79.5)$ & $<0.001$ \\
\hline \multicolumn{5}{|c|}{ Patients with HRQOL PsH score ${ }^{\mathrm{b}}>1 \mathrm{SD}$ above } \\
\hline the mean of healthy children & 43/547 (7.9) & $11 / 51(21.6)$ & $16 / 44(36.4)$ & $<0.001$ \\
\hline Patients with VAS well-being ${ }^{\mathrm{c}}>0$ & $208(37.5)$ & $41(77.4)$ & $40(88.9)$ & $<0.001$ \\
\hline Patients with VAS pain ${ }^{\mathrm{c}}>0$ & $137(24.7)$ & $38(71.7)$ & $31(68.9)$ & $<0.001$ \\
\hline Patients with VAS disease activity ${ }^{\mathfrak{c}}>0$ & $167(30.1)$ & $43(81.1)$ & $39(86.7)$ & $<0.001$ \\
\hline Parent subjective rating of disease status & & & & $<0.001$ \\
\hline Remission & $476 / 543(87.7)$ & $29 / 50(58.0)$ & $12(26.7)$ & \\
\hline Persistent activity & $44 / 543(8.1)$ & $13 / 50(26.0)$ & $18(40.0)$ & \\
\hline Flare & $23 / 543(4.2)$ & $8 / 50(16.0)$ & $15(33.3)$ & \\
\hline $\begin{array}{l}\text { Parents satisfied with their child's illness } \\
\text { outcome }\end{array}$ & $515 / 546(94.3)$ & $45(84.9)$ & $24 / 44(54.5)$ & $<0.001$ \\
\hline
\end{tabular}

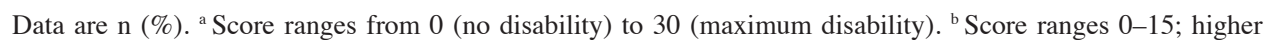
scores indicate worse HRQOL. We previously found that the mean (SD) score of the $\mathrm{PhH}$ and $\mathrm{PsH}$ subscales in 801 Italian healthy children was 0.8 (1.2) and 1.8 (1.7), respectively (Bertamino M, et al. Unpublished data). ${ }^{\mathrm{c}}$ All VAS range from 0 (best) to 10 (worst). CID: clinical inactive disease; MS: morning stiffness; HRQOL: health-related quality of life; $\mathrm{PhH}$ : physical health; PsH: psychosocial health; VAS: visual analog scale.

of CID usually prompts the physician to decrease or even to discontinue the treatments. Concordance with physician assessment of the disease state may facilitate adherence to therapeutic decisions. This limitation was partially amended by inclusion of MS assessment in 2011 CID criteria, which, however, consider MS $\leq 15$ min compatible with the state of CID.
Our results indicate that most parents may not consider their child's disease in remission in the presence of MS, even of a short duration. It should be acknowledged, however, that $58 \%$ of parents of children with MS $\leq 15$ min still judged their child to be in remission and $85 \%$ of them were satisfied with illness outcome. On the other hand, $30 \%$ of parents of children with no MS rated the VAS disease activity $>0$ and 
around $12 \%$ stated that their child had persistent activity or disease flare. Thus, although our findings suggest that the 2011 CID criteria should be modified by stating that no MS should be present, studies in different patient populations and prospective validation are needed before considering this change.

Our study should be interpreted in the light of some caveats. Our findings may not be generalizable because all parents and patients lived in Italy. Parent perception of disease burden has been shown to vary across ethnic and cultural environments ${ }^{11}$. We did not investigate the influence on parent evaluations of non-disease-related factors such as depression, coping strategies, anxiety, and family functioning, or persistent pain symptoms independent of joint inflammation. We recognize that requiring absence of MS for the definition of CID does not imply that a parent report of brief (e.g., $5 \mathrm{~min}$ ) MS is highly sensitive and specific to detect the degree of activation of the inflammatory process in JIA. In the clinic, we frequently see patients who have no inflammatory arthritis whatsoever, yet report brief MS. Likewise, requiring no MS as part of CID would neither prevent patients with brief MS having their treatment "inappropriately" tapered, nor result in patients with $5 \mathrm{~min}$ of MS but no clinical evidence of disease activity as having their antirheumatic medications "appropriately" switched or increased to "reach the target."

We found that the presence of MS in patients with JIA classified as having CID by formal definitions is associated with worse parent perception of child's health and disease status. Our observation indicates that the 2011 CID criteria may be preferred over the 2004 version, and that a change in the former criteria to require absence of MS should be considered.

\section{ACKNOWLEDGMENT}

Permission for use of material derived from the Childhood Health Assessment Questionnaire (CHAQ) and the Child Health Questionnaire (CHQ) is granted through the scientific cooperation of the copyright holder ICORE of Woodside, California, and HealthActCHQ Inc. of Boston, Massachusetts, USA. All CHQ-related inquiries should be directed to licensing@healthactchq.com. All CHAQ-related inquiries should be directed to gsingh@stanford.edu.

\section{REFERENCES}

1. Selvaag AM, Flatø B, Lien G, Sørskaar D, Vinje O, Førre O. Measuring health status in early juvenile idiopathic arthritis: determinants and responsiveness of the child health questionnaire. J Rheumatol 2003;30:1602-10.

2. Passarelli CM, Roizenblatt S, Len CA, Moreira GA, Lopes MC, Guilleminault $\mathrm{C}$, et al. A case-control sleep study in children with polyarticular juvenile rheumatoid arthritis. J Rheumatol 2006;33:796-802.

3. Wallace CA, Ruperto N, Giannini E; Childhood Arthritis and Rheumatology Research Alliance; Pediatric Rheumatology International Trials Organization; Pediatric Rheumatology Collaborative Study Group. Preliminary criteria for clinical remission for select categories of juvenile idiopathic arthritis. J Rheumatol 2004;31:2290-4.

4. Wallace CA, Giannini EH, Huang B, Itert L, Ruperto N; Childhood Arthritis Rheumatology Research Alliance; Pediatric Rheumatology Collaborative Study Group; Paediatric Rheumatology International Trials Organisation. American College of Rheumatology provisional criteria for defining clinical inactive disease in select categories of juvenile idiopathic arthritis. Arthritis Care Res 2011;63:929-36.

5. Petty RE, Southwood TR, Manners P, Baum J, Glass DN, Goldenberg J, et al; International League of Associations for Rheumatology. International League of Associations for Rheumatology classification of juvenile idiopathic arthritis: second revision, Edmonton 2001. J Rheumatol 2004;31:390-2.

6. Verazza S, Negro G, Marafon D, Consolaro A, Martini A, Ravelli A. Possible discontinuation of therapies after clinical remission in juvenile idiopathic arthritis. Clin Exp Rheumatol 2013;4 Suppl 78:S98-101

7. Ravelli A, Viola S, Ruperto N, Corsi B, Ballardini G, Martini A. Correlation between conventional disease activity measures in juvenile chronic arthritis. Ann Rheum Dis 1997;56:197-200.

8. Filocamo G, Davi S, Pistorio A, Bertamino M, Ruperto N, Lattanzi $\mathrm{B}$, et al. Evaluation of 21-numbered circle and 10-centimeter horizontal line visual analog scales for physician and parent subjective ratings in juvenile idiopathic arthritis. J Rheumatol 2010;37:1534-41.

9. Consolaro A, Bovis F, Pistorio A, Cimaz R, De Benedetti F, Miniaci A, et al; Paediatric Rheumatology International Trials Organisation (PRINTO). The Italian version of the Juvenile Arthritis Multidimensional Assessment Report (JAMAR). Rheumatol Int 2018;38 Suppl 1:251-8.

10. Ravelli A, Martini A. Remission in juvenile idiopathic arthritis. Clin Exp Rheumatol 2003;21 Suppl 31:S89-93.

11. Consolaro A, Ravelli A. Defining criteria for disease activity states in juvenile idiopathic arthritis. Rheumatology 2016;55:595-6. 